\title{
121 \\ CSCW, Groupware and Workflow: Experiences, State of Art, and Future Trends
}

\section{Steven Poltrock}

Boeing Information \& Support
Services

PO Box 3707 MS 7L-49

Seattle, WA 98124-2207

USA

steven.poltrock@boeing.com

\author{
Jonathan Grudin \\ Information and Computer Science \\ Department \\ University of California at Irvine \\ Irvine, CA 92717 \\ USA \\ grudin@ics.uci.edu
}

\begin{abstract}
Technology to support groups is rapidly coming into use and having an impact on us, our organizations, and society. This course addresses recent experiences, current possibilities, and future trends and shocks. Lecture and video illustrations are accompanied by discussions in which participants organize and present their collective experiences with groupware and workflow technologies, and CSCW issues and methods. The instructors summarize the current composition of the CSCW community and the state of the art in technology, and organize discussion of fundamental challenges that face us as users (and developers) of these technologies.
\end{abstract}

KEYWORDS Groupware, workflow, computer-supported cooperative work, coordination theory, organizational design, sociotechnical evolution

\section{CSCW, GROUPWARE \& WORKFLOW: DRAWING ON EXPERIENCE}

Five years ago most experience in computer-supported cooperative work was confined to research laboratories. The situation has changed. Although relying primarily on lecture and video illustrations, this tutorial provides a framework for attendees to share and build on their diverse experiences with different types of groupware.

\section{A MULTI-DISCIPLINARY CAST OF THOUSANDS}

We will outline the major contributors to CSCW and groupware: researchers from diverse fields, developers working in varied contexts, and users in different cultures. By reviewing their backgrounds as technologists, behavioral and social scientists, customers, and users, we can see the opportunities to learn from people whose expertise or experience complements our own (see Grudin, 1994a).

\section{THE STATE OF THE ART}

Our survey of the state of the art serves two purposes: It provides information about research and development activity to tutorial participants whose experience or expertise is likely to be focused in one or two areas, and the framework for presenting the technologies serves to focus subgroup discussions of experiences with the 
technologies. We identify three categories of groupware: communication, cooperation, and coordination.

\subsection{Communication Support}

Electronic mail, desktop conferencing, and video conferencing are changing both personal and business communications. Businesses use email to accelerate the flow of information and reduce coordination costs [7]. Video conferencing is used for group communication within geographically distributed companies and between corporate partners. Desktop conferencing focuses communication on work products. Each type of communication groupware offers unique advantages, and new technologies can achieve all the advantages by integrating email and conferencing features.

\subsection{Cooperation Support}

Groupware supports cooperation by enabling interaction through a shared document or collection of documents. For example, document management systems help teams collaborate by providing access and version control, document search, and status tracking. Application development environments customize the structure and functionality of the system to the task. Meeting support technologies enable all participants to collaborate in the production of a shared document or documents. In a brainstorming session all users can enter as many ideas as they want anonymously.

\subsection{Workflow: Support for Coordination}

Businesses are attempting to increase quality and reduce cost by modeling and improving their internal processes. Coordination groupware can capture and coordinate these processes. Work flow management systems model the sequence of subtasks in a work process and the roles performed by each individual. When each subtask is completed, the work is automatically routed to the person responsible for the next subtask.

\section{CHALLENGES IN DESIGN AND EVALUATION}

Successfully overcoming technical hurdles has not guaranteed success. In this section of the tutorial we briefly review behavioral, social, and organizational phenomena that have been observed to undermine technically impressive applications (Grudin, 1994b). Some of these obstruct the development of useful and usable software, some hinder the acquisition of the systems, and many get in the way of successful use. Throughout the tutorial, participants will be invited to identify those challenges that they have encountered or anticipate as they set about acquiring, using, or developing groupware.

\section{OVERCOMING THE CHALLENGES}

The literature includes case studies of successful use of groupware technologies, including some recently conducted by the organizers (see Grudin \& Palen, 1995). In this section, we will briefly review several promising, novel approaches to the successful design and introduction of groupware.

\section{GROUPWARE AND THE FUTURE}

Although we can point to trends and possibilities, we cannot predict which groupware or workflow products will emerge, which will succeed, or which will fail. Nevertheless, at a broader level, we can identify changes that are likely to raise fundamental questions for us as users as well as developers of these technologies. Members of society, informed or otherwise, will have to resolve how these technologies are used. We will conclude the tutorial by identifying a few global developments and invite the participants to identify others. One is the tension between liberating and constraining effects that often accompany technologies, including groupware. Another, perhaps the most fundamental, is the increased visibility of activity in the global village and our reaction to seeing much of that activity for the first time.

\section{REFERENCES}

Grudin, J. (1994a) CSCW: History and focus. IEEE Computer, 27, 5, 19-26.

Grudin, J. (1994b) Groupware and social dynamics: Eight challenges for developers. Communications of the ACM, 37, 1, 92-105.

Grudin, J. and Palen, L. (1995) Why groupware succeeds: Discretion or mandate? Proceedings ECSCW'95 European Computer Supported Cooperative Work Conference. 